\title{
The use of discrete mathematics algorithms in technological design
}

\author{
Nina Glinskaya* and Isabella Belonovskaya \\ Orenburg State University, Orenburg, Russia
}

\begin{abstract}
The article examines the possibility of the formalization of the main stages of a detail mechanical processing using discrete mathematics methods. There are examples of using the graph theory for modeling practically all the stages of the development of processing technology. For structural optimization of technological process it is offered to use the problem of the traveling salesman. The choice of technological bases may be carried out both with using the graph theory and the set theory. The article makes a conclusion about the effectiveness of discrete mathematics methods for the formalization of decision making processes during technological processing.
\end{abstract}

Modern development of machine-building production is impossible without using automated systems of technological preparation of the production (ASTPP). One of the leading parts in ASTPP is played by the systems of automated projecting technological processes, known as CAPP. Most of CAPP which are presented today at the market realize the dialogue projecting regime, when all the technological decisions are made by a man, and a projecting systems forms different documents automatically. A low level of automation taking of technological decisions to a certain degree is connected with an extremely low level of formalization of technological decision making. One of the variants of solving the problem of technological projecting formalization is attracting mathematical methods of technological problems description. The intermittence of the mechanical treatment process causes using discrete mathematics methods.

One of the most claimed sections of discrete mathematics is graph theory. It is the most powerful means in solving the problem of formalization of different stages of technological process working out.

A graph is an aggregate of not empty set of vertexes and a number of edges or arcs. To solve the majority of technical projecting problems the graph vertexes will mark the detail surfaces. Detail surfaces must be marked in letters or in figures. Edges or arcs will describe the surfaces intercommunications depending on the problem being solved.

The graph may be used beginning with one of the first technological projecting stages - the study and analyses of the detail draft. To do it the dimensional relationships graph is made. In this graph the vertexes describe the detail surface, and the edges describe connecting them dimensions.

\footnotetext{
* Corresponding author: $\underline{n} \_$u_gl $@$,rambler.ru
} 
Using the connectedness according to which any two graph vertexes may be connected by a chain, it is possible to analyze the quality of dimensions putting. If the dimensions connections graph is coherent, therefore the dimensions are put correctly. (Figure 1-4).
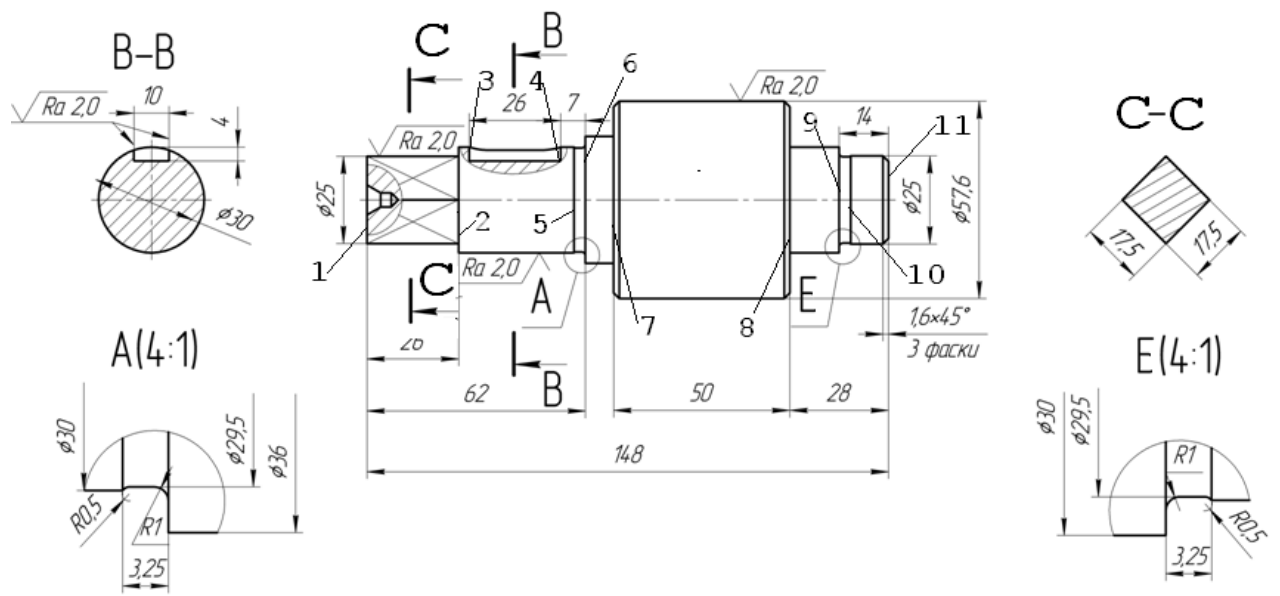

Fig. 1. Drawing of the shaft with correct sizing

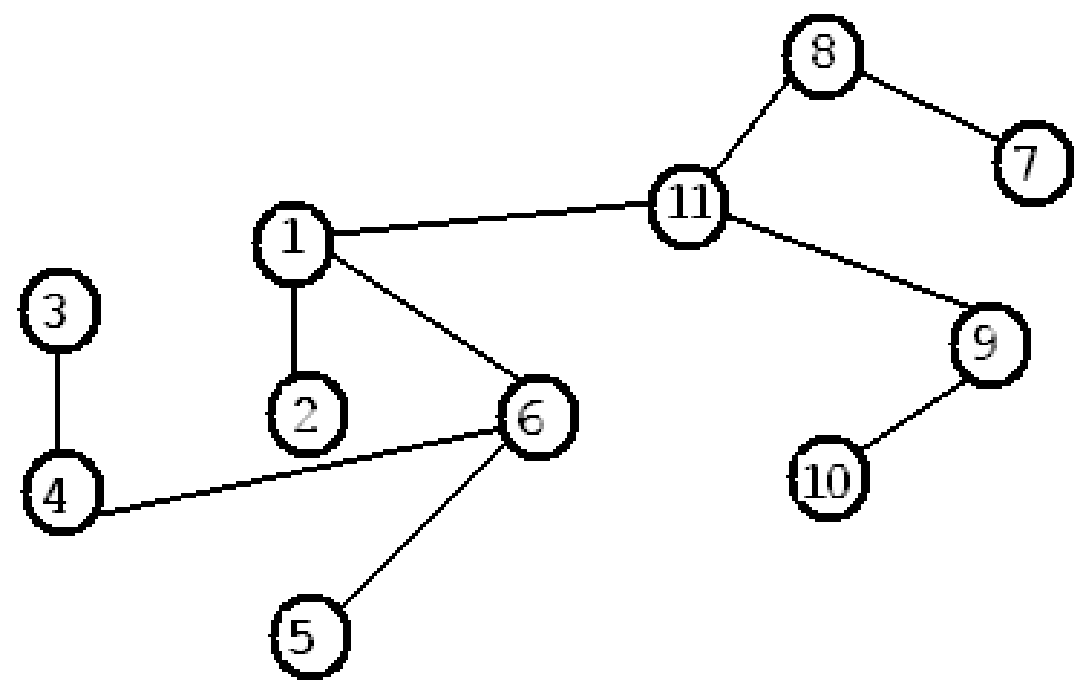

Fig. 2. Connected graph of dimensional relationships 


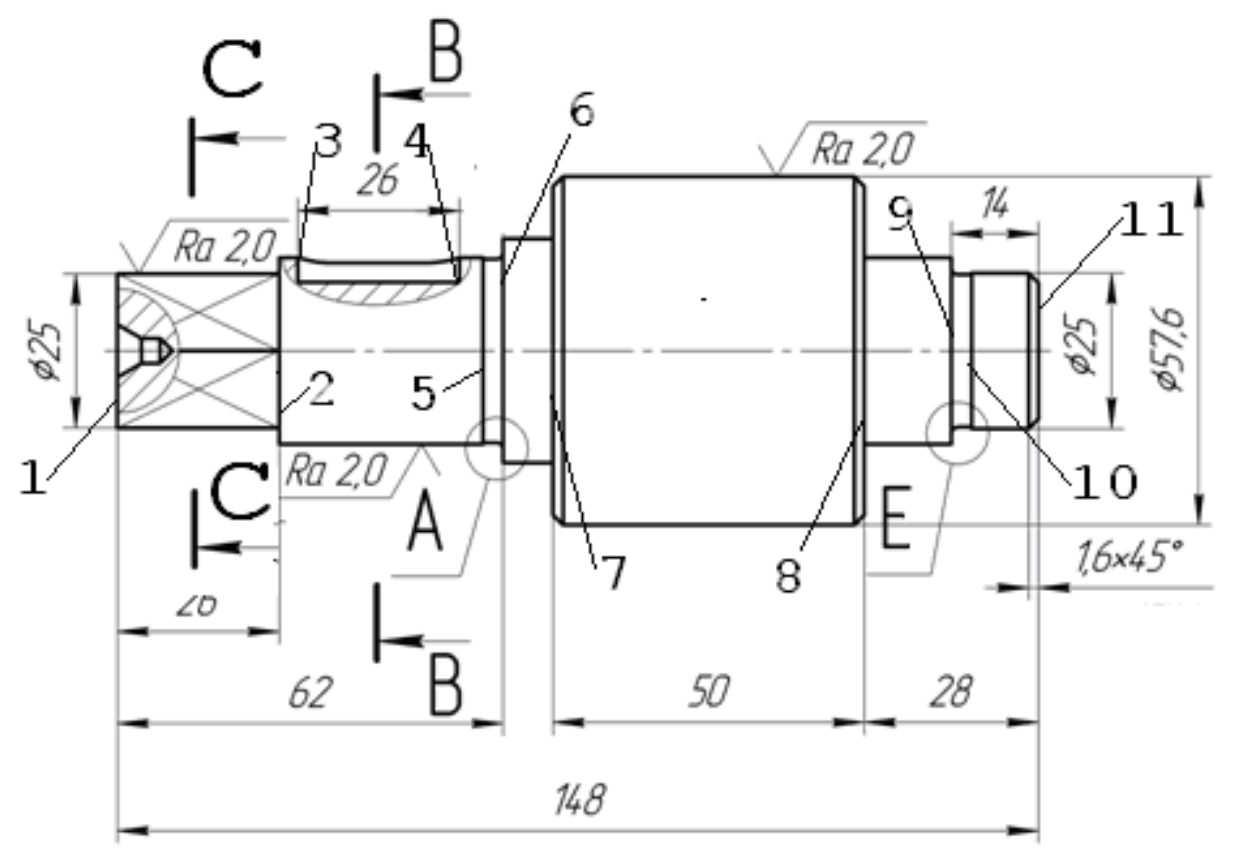

Fig. 3. The drawing shaft with a missing size

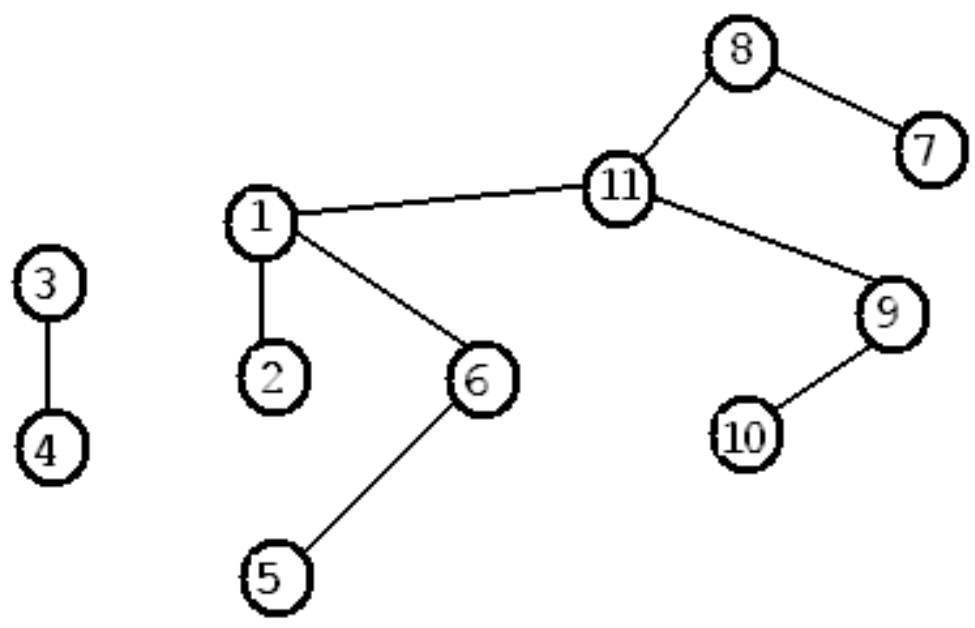

Fig. 4. Non connected disjoint graph of dimensional relationships

The next stage, when it is possible to use the graph theory, is the choice of technological bases. To solve this problem is also possible with using dimensional connections graph, and not only visually, but also using the matrix describing the graph. Such matrix is called a connectivity vertexes matrix. In conformity with machine building technology this matrix can be named the dimensional connection matrix. At the crossing of the line and the column which signify the graph vertexes one is put, if indicated surfaces are connected with dimensions or the demand of mutual disposition and zero is put, in other wise. It is known from the basing theory that it is desirable to use the surfaces concerning which the greatest number of dimensions is set as technological bases. So, it is necessary to determine the number of ones in every line. As every line means the graph vertex with which the detail 
surfaces is connected, the lines with the greatest number of ones will show the surfaces with the greatest dimensions connections number. Therefore just these surfaces are preferably used as technological bases. Demands of relative surfaces position are often set besides dimensions. During choosing technological bases the most influence is exerted not so much on the controlled dimensions as on the demand of relative surfaces positions. For the calculation of this factor it is possible to put ones for marking the dimensions and the figure two for marking demands of surfaces position to dimensions connections matrix. In this case during the dimensions connections matrix analysis the line elements sum is defined. Such method was realized during solving the problem of bases choice automation [1].

In the cases stated above the main theses of graph theory are used. If we examine algorithms, one of the most popular in the practice of machine building technological process modeling is the group of the way search algorithms on the graph or algorithms of the vertexes graph round.

In this algorithms group the so-called problem of "traveling salesman" is of the greatest interest. This algorithm can be used during technological process structural optimization. Mathematically the problem of the "traveling salesman" can be formulated in the following way [2-5 ].

"There are $\mathrm{N}$ towns. Living the initial town A1 the traveling salesman must visit all the towns once and return to the town A1. The problem is in defining the succession of visiting the towns, with help of which the traveling salesman has to minimize some efficiency criterion: driving cost, driving time, summary distance."

For costing there is a matrix of conditions $\mathrm{C}$, containing investment for moving from every town to every one, taking into consideration that it is possible to move from any town to any one, expect for just the same one (there is no diagonal in the matrix). The idea of solution is finding the route answering all conditions and having the minimum expenditure. For formal formulation of the problem the towns are numbered $\left(\mathrm{j}_{1}, \mathrm{j}_{2}, \ldots, \mathrm{j}_{\mathrm{n}}, \mathrm{j}_{1}\right)$, and all $\mathrm{j}_{1}$.. $\mathrm{j}_{\mathrm{n}}$ - are different numbers $\mathrm{j}_{1}$ repeating at the beginning and at the end shows that the rearrangement is caught in cycles. Distances between pairs of towns $\mathrm{C}_{\mathrm{ij}}$ from matrix $\mathrm{C}$. The problem to find such a route that can minimize the functional

$$
L=L(t)=\sum_{k=1}^{n} C_{i k} C_{i k+1}
$$

Let us examine using this problem after the example of optimization of the working of the equipment idling during machining or optimized controlling the detail holes of the same type.

Imagine that it is necessary to make centering 10 holes before drilling or to control the quality of machining a group of holes (figure 5). If in formulating the problem of a "traveling salesman" to substitute the word "town" for the word "hole" it will be a mathematical statement of the problem of optimization of the working parts of the equipment idling during machining or controlling the hole groups.

There are many different algorithms of this problem solution, both approximate and exact, and the range of values may be essential [ 6 ]. One of exact algorithms is the "branch - and - bound method" known also as Little algorithm. The main point of the algorithm consists in fact that at first "lower estimate" is defined, that is the minimal possible cost or the length of the route. For this reason minimal elements are subtracted from every row and column and their sum is defined. 


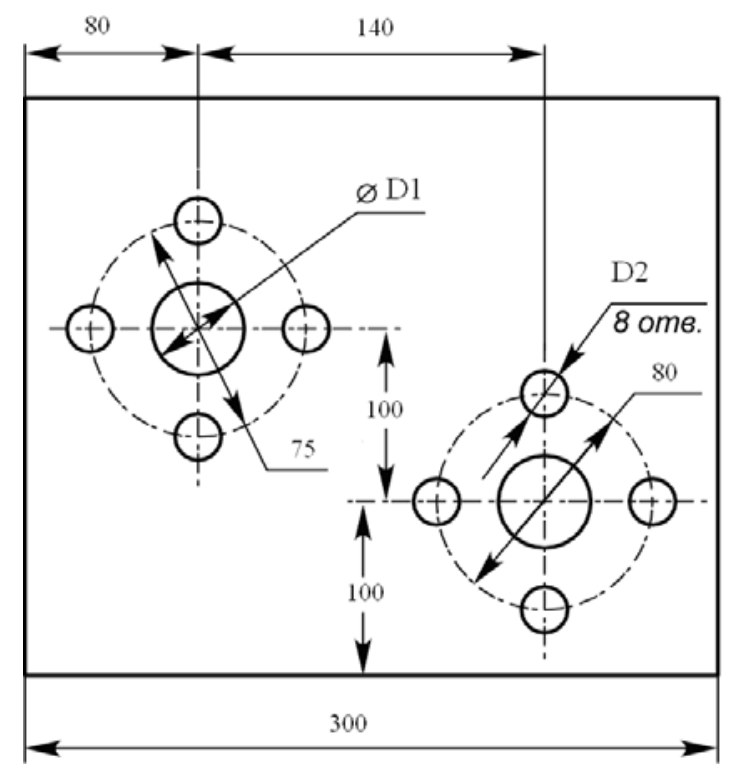

Fig. 5. The example of the location of processed or controlled.

This action is called matrix reduction by rows and columns. The lesser length rout doesn't exist. As a result of the reduction, minimum one zero element is formed in every row and every column. If make a route according those zero elements, we'll have an optimal variant. To choose a variant the zero estimation procedure is used, which consists of summing up minimal elements of the row and column, containing an estimated zero. Little recommended to use zeroes with maximum estimation. For prohibition loop the prohibition for mirror elements is introduced, for example, if variant " $1-2$ " is used, it's impossible to us "2-1". As a result of such prohibitions rows and columns without zeroes can be formed. In this case matrix reduction is maid again and subtracted quantity is added to the "lower estimate". Using this algorithm in this problem we got the result shown in Figure 6.

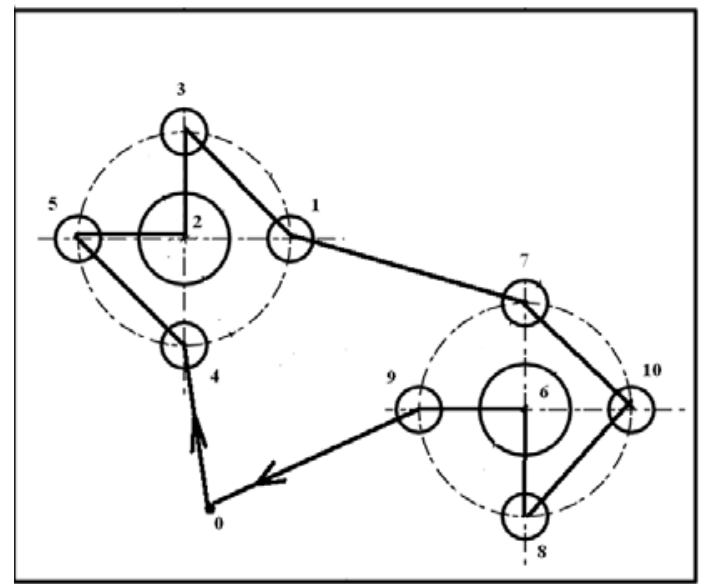

Fig. 6. The reference points walk according to the branch - and - bound method

The next discrete mathematics section, which may be successfully used for the formalization of the technological designing main stages, is the set theory. And the set concept modification, offered by V. Pavlov [7], which consists of adding different 
properties to set elements. It is offered to mark properties by colors by analogy with the colored graphs. In this case the set is described not with help of a line with the enumeration of elements, but with the help of the matrix, its lines describe set elements and it columns describe the properties. The example of using such modified sets may be solving the problems about the bases choice. The set elements are the surfaces of the details which may have the following properties: the size, connected with the surface, the technical requirement in relation to the surface, the surface length along one coordinate axis, the surface length along the second coordinate axis. During the analysis of the matrix lines it is possible to determine not only which surfaces may be bases, but what kind of bases they are by the degrees of freedom deprived.

The analysis of taking decision methods during the detail machining shows their weak formalization, which restrains the CAPP systems development. The algorithms and discrete mathematics provisions given in the article may be used quite successfully for formalization of the taking technological decisions processes. The formalized processes may be automated more easily; therefore using discrete mathematics algorithms in practice of technological designing will make it possible to raise the level of CAPP systems automation.

\section{References}

1. Glinskaya N Y Materials Science and Engineering. - Vol. 709, Iss. 4 P. 1-4 (2020).

2. Glinskaya N Y, Elagin V V Journal of Physics. -. - Vol. 1333, Iss. 4. (2019)

3. Novikov F A Discrete mathematics for computing St.Petersburg: Piter, 2000 267-268

4. Miranda P A, Blazquez C A, Obreque C, Maturana-Ross J and Gutierrez-Jarpa G European Journal of Operational Research 271(3) 1014-1036 (2018)

5. Pansart L, Catusse N and Cambazard H Computers and Operations Research 100117 127 (2018)

6. Silva A A, Morabito R and Pureza V Expert Systems with Applications 112 321-330 (2018)

7.V. V. Pavlov CALS technologies in mechanical engineering (mathematical models) Moscow: MSTU STANKIN (2002) 